Original article

\title{
Morphofunctional changes in the adrenal glands of juvenile rats systematically exposed to hypergravity
}

\author{
Gennady A. Moroz, Maxim A. Kriventsov, Sergey A. Kutia \\ V.I. Vernadsky Crimean Federal University, Simferopol, Russia
}

Received 7 January 2018, Revised 26 August 2018, Accepted 31 August 2018

(C) 2018, Moroz G.A., Kriventsov M.A., Kutia S.A.

(C) 2018, Russian Open Medical Journal

\begin{abstract}
Aim of this study was to assess morphofunctional transformations in the adrenal glands of juvenile rats systematically exposed to + Gx acceleration.

Material and Methods - Rats in the experimental group were exposed to transversely-directed gravitational overloads (9 G) produced by experimental centrifuge C-2/500 (three times, each time 3 minutes exposure with 30 seconds break between sets). Microscopical, histomorphometric (thickness of cortical zones; cortex-medulla ratio; absolute and relative area of zona glomerulosa, zona fasciculate, zona reticularis, and medulla; number and density of cells in each zone; area of nuclei, cytoplasm and liposomes in endocrinocytes), ultrastructural and statistical methods were used.

Results - Structural transformations found in adrenal glands are adaptive responses to stress and hemodynamic changes caused by systematic influence of gravitational overloads. The expressed hemodynamic changes combined with stress-induced morphofunctional transformations, manifested as signs of functional stress of cortical and medullar endocrinocytes on the background of dystrophic and destructive ultrastructural changes were revealed. Increase of number of exposures to 45 leads to further stabilization of morphofunctional condition of adrenal glands.

Conclusion - Structural transformations and features of the functional tension of the cortical and medullary endocrinocytes are limited as "systemic structural trace".
\end{abstract}

Keywords: morphology, rats, adrenal glands, hypergravity

Cite as Moroz GA, Kriventsov MA, Kutia SA. Morphofunctional changes in the adrenal glands of juvenile rats systematically exposed to hypergravity. Russian Open Medical Journal 2018; 7: e0401.

Correspondence to Maxim A. Kriventsov. Address: Lenin Boulevard 5/7, Simferopol, 295000, Russia. Phone: +7-978-7092328. E-mail: maksimkgmu@mail.ru.

\section{Introduction}

The study of the adaptation of the organism to external factors, including gravitational overloads, is an actual medical and biological problem. Overloads cause hemodynamic changes and respiratory disturbances as well as lead to a complex of physiological reactions known as "general adaptation syndrome" $[1,2]$. Like any impact, hypergravity has a certain dose-dependent effect. The overload magnitude, duration, repeatability, and vector result in adaptation, activation and increasing of resistance or adaptation failure and decreasing of organism resistance not only to this factor but also to another one's [3-6].

One of the leading roles in the implementation of adaptive reactions of the body is played by the endocrine system and adrenal glands, in particular, which closely interacting with nervous and immune systems regulate metabolic and energy processes $[7,8]$. Meanwhile, in the literature there are practically no data on the effect of repeated gravitational overloads on the structural transformations occurring in the adrenal glands.

The aim of this study was to assess morphofunctional transformations in the adrenal glands of rats of juvenile age exposed to hypergravity.

\section{Material and Methods}

36 male Wistar rats (initial weight $120-130 \mathrm{~g}$, initial age 2 months) were randomly divided into two groups: control (C) $(n=18)$ and experimental (G) $(n=18)$. According to experiment duration $(10,30$, and 45 days) they were subdivided into three subgroups ( $n=6$ in each).

Rats in the experimental group were exposed to transverselydirected gravitational overloads from front to back (+Gx) (9 G) produced by experimental centrifuge $\mathrm{C}-2 / 500$ (three times, each time 3 minutes exposure with 30 seconds break between sets). It had an arm length of $0.5 \mathrm{~m}$ and was capable of producing acceleration range from 1 to $50 \mathrm{~g}$. The onset rate was $1.4-1.6 \mathrm{~g} / \mathrm{s}$, offset rate $-0.6-0.8 \mathrm{G}$ per second. Each rat was placed inside a cylindrical plastic device beforehand and after that in the box at centrifuge arm. The rats in the control group were mounted on the surface of running centrifuge. All experiments were conducted every day at the same time.

Rats were anesthetized with thiopental sodium and decapitated next day after last exposure. All procedures performed in studies involving animals were in accordance with the ethical standards of the institution or practice at which the studies were conducted. 
Table 1. The ratio (\%) of the adrenal cortex zones in juvenile rats under the systematic exposure to +Gx acceleration (M $\pm m$ )

\begin{tabular}{|c|c|c|c|c|c|}
\hline \multirow{2}{*}{ Experimental group } & \multirow{2}{*}{ Adrenal medulla } & \multirow{2}{*}{ Adrenal cortex } & \multicolumn{3}{|c|}{ Zone of the adrenal cortex } \\
\hline & & & Glomerulosa & Fasciculata & Reticularis \\
\hline \multicolumn{6}{|c|}{10 days } \\
\hline $\mathrm{C}$ & $12.60 \pm 0.23$ & $87.40 \pm 1.15$ & $9.17 \pm 0.38$ & $69.01 \pm 0.97$ & $21.82 \pm 0.53$ \\
\hline G & $5.49 \pm 0.24 *$ & $94.51 \pm 1.09 *$ & $10.07 \pm 0.43$ & $77.30 \pm 0.93 *$ & $12.63 \pm 0.61^{*}$ \\
\hline \multicolumn{6}{|c|}{30 days } \\
\hline $\mathrm{C}$ & $9.89 \pm 0.22$ & $90.11 \pm 1.52$ & $9.27 \pm 0.13$ & $76.01 \pm 1.57$ & $14.72 \pm 0.41$ \\
\hline G & $7.34 \pm 0.24 *$ & $92.66 \pm 0.75$ & $8.02 \pm 0.15^{*}$ & $80.78 \pm 1.02 *$ & $11.21 \pm 0.54^{*}$ \\
\hline \multicolumn{6}{|c|}{45 days } \\
\hline C & $11.34 \pm 0.18$ & $88.66 \pm 0.18$ & $11.84 \pm 0.52$ & $77.31 \pm 0.92$ & $10.85 \pm 0.40$ \\
\hline G & $12.40 \pm 0.33^{*}$ & $87.60 \pm 0.70$ & $16.27 \pm 0.58^{*}$ & $71.25 \pm 1.45^{*}$ & $12.48 \pm 0.56^{*}$ \\
\hline
\end{tabular}

$*-p<0.05$ compared to the control.

Table 2. Nucleus area $\left(\mu \mathrm{m}^{2}\right)$ of adrenal endocrinocytes in juvenile rats under the systematic exposure to $+\mathrm{Gx}$ acceleration $(\mathrm{M} \pm \mathrm{m})$

\begin{tabular}{|c|c|c|c|c|}
\hline \multirow{2}{*}{ Experimental group } & \multicolumn{3}{|c|}{ Zone of the adrenal cortex } & \multirow{2}{*}{ Adrenal medulla } \\
\hline & Glomerulosa & Fasciculata & Reticularis & \\
\hline \multicolumn{5}{|c|}{10 days } \\
\hline $\mathrm{C}$ & $31.33 \pm 0.47$ & $32.45 \pm 0.42$ & $31.86 \pm 0.77$ & $22.17 \pm 0.36$ \\
\hline G & $23.99 \pm 0.45^{*}$ & $37.03 \pm 1.25^{*}$ & $36.95 \pm 1.26^{*}$ & $23.24 \pm 0.63^{*}$ \\
\hline \multicolumn{5}{|c|}{30 days } \\
\hline C & $17.37 \pm 0.21$ & $21.44 \pm 0.13$ & $18.85 \pm 0.80$ & $23.08 \pm 0.22$ \\
\hline G & $16.05 \pm 0.55^{*}$ & $23.17 \pm 0.86$ & $22.35 \pm 0.68 *$ & $23.11 \pm 0.01$ \\
\hline \multicolumn{5}{|c|}{45 days } \\
\hline C & $9.33 \pm 0.31$ & $23.22 \pm 0.17$ & $22.00 \pm 0.56$ & $25.54 \pm 0.33$ \\
\hline G & $13.19 \pm 0.38 *$ & $19.33 \pm 0.39 *$ & $16.40 \pm 0.60 *$ & $19.06 \pm 0.48^{*}$ \\
\hline
\end{tabular}

$*-p<0.05$ compared to the control.

The left adrenal gland was extracted and weighed (grams). The determination of the organ weight was carried out using an analytical balance AXIS AN500 (capacity 0.01-50.0 g, reading unit $0.1 \mathrm{mg}$ ). The organ-to-body weight ratio was calculated as well.

For microscopic analysis, the adrenal glands were fixed in $10 \%$ neutral formaldehyde, dehydrated in increasing concentrations of ethanol, cleared in xylene, and embedded in paraffin. Serial sections $(4-6-\mu \mathrm{m})$ prepared by sliding microtome were stained with hematoxylin and eosin (H\&E), van Gieson stain. Lipids were revealed by sections stained with Sudan black B as well as by semithin one's preliminarily fixed in osmium tetroxide. We used capture equipment consisting of microscope Olympus CX 31 coupled to video camera Olympus C5050. The following objectives were used: Plan 4x oo/-, Plan 10x /0.25, Plan 20x /0.40, Plan 40x /0.65, Plan 100x /0.17. Images were obtained using several magnifications. Zoom 173 was used as objective adjustment. Obtained in this way digital images are TIFF files with color depth 24 bit.

Histomorphometry was performed using the software ImageJ (U.S. National Institutes of Health, Bethesda, Maryland, USA) [9]. Measurements included thickness of cortical zones; cortexmedulla ratio; absolute and relative area of zona glomerulosa, zona fasciculate, zona reticularis, and medulla; number and density of cells in each zone; area of nuclei, cytoplasm and liposomes in endocrinocytes.

For transmission electron microscopy just after taking of tissues, $1 \mathrm{~mm}^{3}$ samples were fixed using $2.5 \%$ glutaraldehyde in $0.1 \mathrm{M}$ phosphate buffer $(\mathrm{pH}=7.2-7.4)$ at $+4^{\circ} \mathrm{C}$. Subsequently, the specimens were postfixed in $1 \%$ osmium tetroxide in same buffer for $1 \mathrm{~h}$. The specimens were then dehydrated through a graded series of ethanols, then acetone for 15 min twice and treated with 1:1 acetone : resin mixture for 2 hours. Further embedding was carried out as following: mixture $A(2.5 \mathrm{ml})+$ mixture $B(2.5 \mathrm{~mL})+$ accelerator DMP-30 (4 drops). Mixture A: epon-812 - $62 \mathrm{~mL}$, dodecenyl succinic anhydride - $100 \mathrm{~mL}$; mixture B: epon-812 - 100 $\mathrm{mL}$, MNA - $89 \mathrm{~mL}$. The polymerization capsules were incubated at $56^{\circ} \mathrm{C}$ for 36 hours.

Epon 812-embedded sections $(1 \mu \mathrm{m})$ prepared by UMPT-7 ultramicrotome (Electron, Sumy, Ukraine) were stained with toluidine blue for selecting of the most appropriate areas for ultrathin sectioning. Ultrathin sections $(30-60 \mathrm{~nm})$ were contrasted with $1 \%$ uranyl acetate and lead citrate, evaluated and photographed using a PEM-125K (Electron, Sumy, Ukraine) transmission electron microscope (TEM).

Statistical data were analyzed using MS Excel (Microsoft, USA) and Statistica 10.0 (Statsoft, USA). Data distribution was normal (according to the results of Kolmogorov-Smirnov test [10]). Thus, parametric statistical methods were used such as arithmetic mean and standard error of mean (SEM) - M $\pm m$. Values were compared by Student's t-test. For all statistical evaluation, a probability of value $p<0.05$ was considered significant (indicated in text as ${ }^{*}$ ).

\section{Results}

Systematic exposure of juvenile rats to $+G x$ acceleration during 10 days results in significant (by 2.3 times) increase in the relative weight of the adrenal glands compared to the control.

It is noted that the relative area of the cortex is increased by $8.14 \%(p<0.05)$ (Table 1$)$ and the relative area of the medulla is decreased by $56.43 \%(p<0.05)$ compared to the control data.

Adrenal parenchyma and stroma are characterized by the pronounced signs of hemodynamic disturbances in the form of expansion and hyperemia of the blood vessels, stasis and sludgephenomenon, as well as diapedesis of red blood cells (RBCs). Connective tissue capsule appears swollen and teased, and in some areas subcapsular edema results in detachment of the capsule from the parenchyma. 


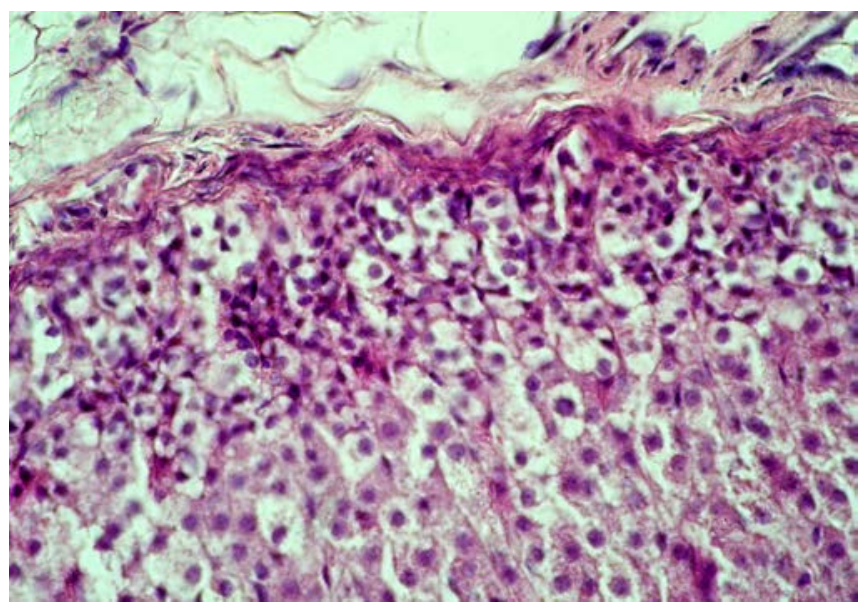

Figure 1. Swelling and teasing of the capsule and loss of integrity of the adrenal zona glomerulosa in juvenile rat under the systematic exposure to 10 -day $+G x$ acceleration. H\&E. $x 400$.

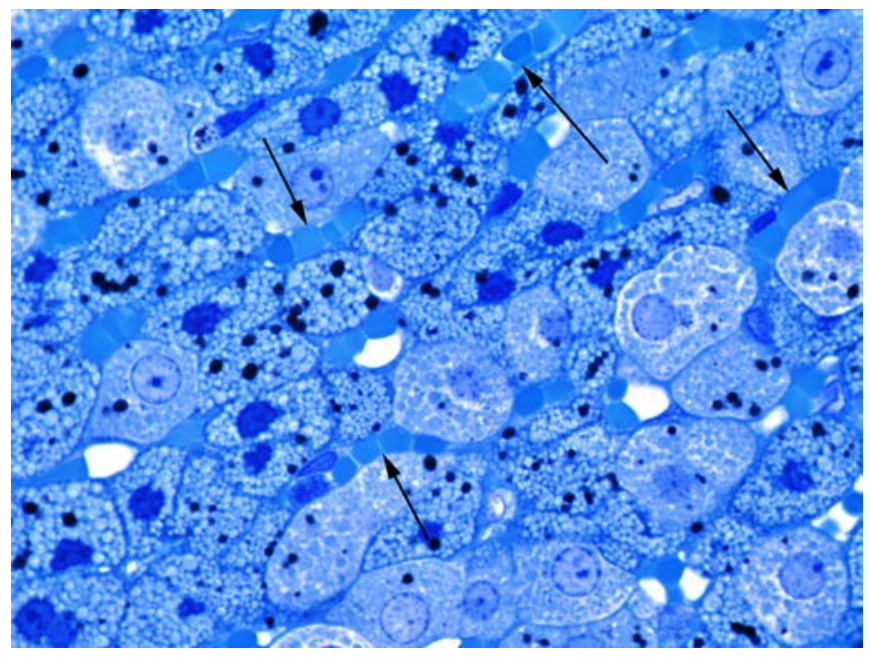

Figure 2. Stasis and sludge of RBCs (arrows) in the capillaries of the zona fasciculata of adrenal cortex in juvenile rat under the systematic exposure to 10-day $+G x$ acceleration. Semithin section. Toluidine blue. x1000.

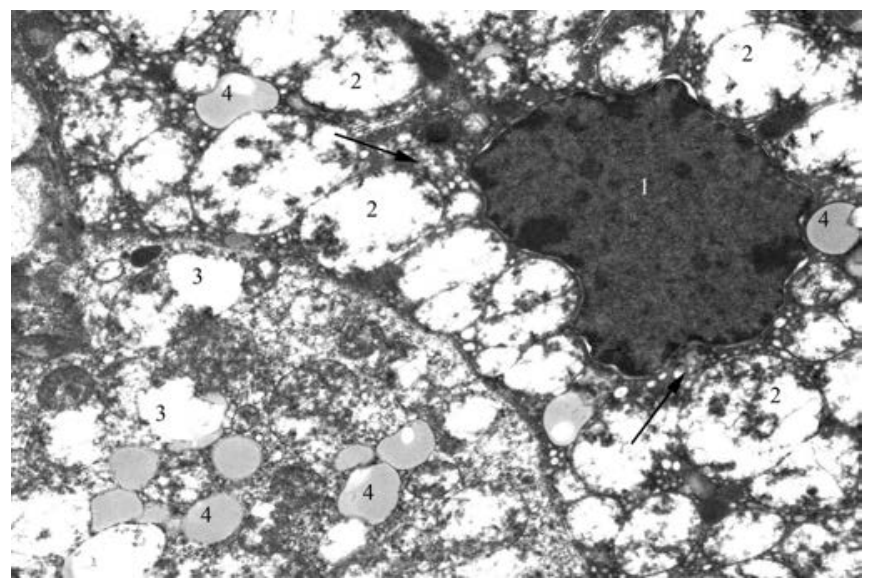

Figure 3. Dark corticocyte in zona fasciculata of the adrenal cortex in juvenile rat under the systematic exposure to 10 -day $+G x$ acceleration. TEM. x6000. 1 - nucleus; 2 - mitochondria; 3 - vacuole; 4 - liposomes; arrows - agranular endoplasmic reticulum.
Zona glomerulosa of the adrenal cortex is characterized by the swelling, losing compactness and loss of the structural properties. Some glomeruli have fuzzy borders. Structural disturbances are accompanied by the shift of some arcuate endocrinocyte clusters deep into zona fasciculata. Such clusters are surrounded by newly formed connective tissue fibers (Figure 1). Adrenocortical cells are characterized by unevenly stained cytoplasm contained shifted to the periphery nucleus of irregular shape. Mean nucleus area decreases by $23.42 \%(p<0.05)$ compared to the control data (Table 2).

Zona fasciculata is expanded with increase in its relative area by $12.02 \%(p<0.05)$ compared to the control. Spongiocytes lose the arrangement. Edema of the connective tissue and activation of fibroblasts can be seen. Capillaries between epithelial strands are plethoric and characterized by the signs of stasis and sludge of RBCs (Figure 2).

Both dark and light spongiocytes are visualized, however, light cells dominates and their relative number (in \%) increases compared to the control by 2.9 times $(p<0.05)$ (Table 3). Although, lightening and enlargement of spongiocytes nuclei is seen (Table 2), the relative nucleus area in adrenal cortex cells decreases by $28.61 \%(p<0.05)$ compared to the control.

The nuclei are displaced to the periphery of the cell with expanded perinuclear space. Karyolemma contains a large number of pores. Cytoplasm is enlightened due to the expanded agranular endoplasmic reticulum, as well as due to small and medium-sized vacuoles, which are formed as a result of the destruction of vesicular mitochondria cristae. Also, the number of mitochondria decreased. Number of lipid droplets with medium and high electron density increases in the cytoplasm, and relative area of liposomes rises by nearly 2.8 times $(p<0.05)$ compared to the control (Table 4$)$.

Most nuclei of dark adrenocorticocytes have an irregular shape due to multiple invaginations of nucleus membrane and contain eccentrically located prominent nucleolus. Heterochromatin in the form of lumps condenses mainly near the inner part of the nuclear membrane. Elements of the endoplasmic reticulum look a little expanded. Large amount of free ribosomes can be seen. Compared to the control, mitochondria are more numerous, and many of them have an oval shape and a lot of vacuoles due to the pronounced edema of matrix and typically complete degradation of the cristae (Figure 3). Mitochondria are characterized by both partial and complete destruction of the cristae, leading to vacuolization appearance. Liposomes contained various in electronic density substance are unevenly located in the cytoplasm, but their number is fewer than in light adrenocorticocytes.

The border between zona fasciculata and reticularis is less pronounced. The last one looks constricted, and its relative area decreases by $42.1 \%(p<0.05)$ compared to the control. Capillaries are over filled with RBCs. There is desquamation of the endothelium and sludge of RBCs in the lumen of some vessels. Endocrinocytes possess ultrastructural manifestations of dystrophic and destructive changes on the background of the signs of synthetic and energy-producing function activation. The nuclear area of such cells increases in comparison with the control by $15.99 \%(p<0.05)$ (Table 2).

Adrenal medulla is characterized by swelling of the intercellular substance, pronounced hyperemia and expansion of the microvasculature bed. The sinusoids are with the pronounced dyscirculatory changes such as stasis and sludge of erythrocytes (Figure 4). The lumen of some vessels has an impaired endothelial lining and clots, accompanied with petechial hemorrhages in the stroma. 
Table 3. The ratio (\%) of dark and light spongiocytes in adrenal zona fasciculata in juvenile rats under the systematic exposure to $+\mathrm{Gx}$ acceleration ( $\mathrm{M} \pm \mathrm{m}$ )

\begin{tabular}{|c|c|c|c|}
\hline Experimental group & Dark spongiocytes & Light spongiocytes & Number of cells per $4000 \mu \mathrm{m}^{2}$ \\
\hline \multicolumn{4}{|c|}{10 days } \\
\hline C & $73.95 \pm 1.28$ & $26.05 \pm 1.28$ & $18.58 \pm 0.46$ \\
\hline G & $24.66 \pm 1.20 *$ & $75.34 \pm 1.20 *$ & $18.58 \pm 0.80$ \\
\hline \multicolumn{4}{|c|}{30 days } \\
\hline C & $63.65 \pm 1.60$ & $36.35 \pm 1.60$ & $11.42 \pm 0.43$ \\
\hline G & $60.15 \pm 2.23$ & $39.85 \pm 0.84$ & $15.00 \pm 0.69 *$ \\
\hline \multicolumn{4}{|c|}{45 days } \\
\hline C & $54.72 \pm 0.75$ & $45.28 \pm 0.90$ & $12.67 \pm 0.37$ \\
\hline G & $55.26 \pm 1.25$ & $44.74 \pm 0.66$ & $12.98 \pm 0.28$ \\
\hline
\end{tabular}

$*-p<0.05$ compared to the control.

Table 4. Relative area (\%) of spongiocyte ultrastructures in adrenal zona fasciculata in juvenile rats under the systematic exposure to $+G x$ acceleration (M土m)

\begin{tabular}{|c|c|c|c|c|}
\hline Experimental group & Type of spongiocyte & Nucleus & Cytoplasm & Liposomes \\
\hline \multicolumn{5}{|c|}{10 days } \\
\hline \multirow[t]{2}{*}{ C } & Light & $21.46 \pm 1.02$ & $78.54 \pm 1.80$ & $2.92 \pm 0.08$ \\
\hline & Dark & $14.63 \pm 0.52$ & $85.37 \pm 2.47$ & $4.64 \pm 0.21$ \\
\hline \multirow[t]{2}{*}{ G } & Light & $15.32 \pm 0.38 *$ & $84.68 \pm 0.81^{*}$ & $8.08 \pm 0.38 *$ \\
\hline & Dark & $11.95 \pm 0.37 *$ & $88.05 \pm 0.89$ & $6.86 \pm 0.34 *$ \\
\hline \multicolumn{5}{|c|}{30 days } \\
\hline \multirow[t]{2}{*}{ C } & Light & $10.84 \pm 0.45$ & $89.16 \pm 2.35$ & $5.05 \pm 0.23$ \\
\hline & Dark & $7.67 \pm 0.38$ & $92.33 \pm 1.62$ & $5.36 \pm 0.25$ \\
\hline \multirow[t]{2}{*}{ G } & Light & $18.38 \pm 0.77^{*}$ & $81.62 \pm 2.31^{*}$ & $4.96 \pm 0.15$ \\
\hline & Dark & $9.95 \pm 0.44 *$ & $90.05 \pm 1.38$ & $7.10 \pm 0.30^{*}$ \\
\hline & & & & \\
\hline \multirow[t]{2}{*}{$C$} & Light & $13.75 \pm 0.57$ & $86.25 \pm 1.76$ & $5.70 \pm 0.27$ \\
\hline & Dark & $9.94 \pm 0.22$ & $90.06 \pm 1.19$ & $6.57 \pm 0.31$ \\
\hline \multirow[t]{2}{*}{$\mathrm{G}$} & Light & $11.97 \pm 0.37^{*}$ & $88.03 \pm 1.64$ & $6.06 \pm 0.22$ \\
\hline & Dark & $8.54 \pm 0.39 *$ & $91.46 \pm 1.39$ & $6.94 \pm 0.35$ \\
\hline
\end{tabular}

$*-p<0.05$ compared to the control.

Chromaffin cells are characterized by somewhat larger than in control irregular nuclei, containing one prominent rounded nucleolus. Cytoplasm of these cells contains small and mediumsize vacuoles formed due to the destruction of mitochondrial cristae and granular endoplasmic reticulum. Endoplasmic reticulum canals are somewhere degranulated. There is a lot of free ribosomes and small secretory granules of medium and high electron density. Moreover, epinephrine-producing cells have more pronounced changes than norepinephrine-producing cells.

30-day exposure to $+G x$ acceleration results in increase in the relative weight of the adrenal glands by more than 2.5 times (compared to the control; $\mathrm{p}<0.05$ ). Morphometric study reveals mild and not statistically significant $(2.83 \%)$ increase in the relative area of the cortex compared to the control (Table 1). Circulatory disturbances are less pronounced than in the previous (10-day exposure) series. Most vessels are dilated, with margination of WBCs in some of them. Same as after 10-day experiment, swelling and delamination of the capsule can be seen. Newly formed connective tissue strands accompanied with vessels spread into the parenchyma up to the zona reticularis.

Zona glomerulosa looks narrower, and quantitatively its relative area decreases by $13.50 \%$ compared to the control $(p<0.05)$. Connective tissue delimited glomeruli is well visualized. Endocrinocytes have tight contact with each other, and their number increases per conditional unit of area. At the same time, adrenocortical nuclei area reduces and becomes less than control values (by $7.62 \% ; \mathrm{p}<0.05$ ) (Table 2). Relative area of zona fasciculata increases by $6.27 \%(p<0.05)$. Radial connective tissue strands with a lot of fiber components clearly delimitate bands of tightly connected adrenocorticocytes. The density of the cell population in this zone increases by $31.39 \%$ compared to the control $(p<0.05)$. However, in contrast to the 10 -day experiment, dark spongiocytes (Figure 5) are predominant in the cell population.

The relative amount of light cells, compared with the control, increases slightly (9.62\%) and such increment is not statistically significant (Table 3). Parallel to this, the absolute and relative areas of the nuclei both dark and light adrenocortical cells significantly increase (Tables 2 and 4). The cytoplasm of dark spongiocytes contains more lipid droplets than in the same cells of the previous experimental series and control. The relative area of the liposomes in the cytoplasm of the dark cells increases by $32.31 \%$ compared to the control $(p<0.05)$. At the same time, light endocrinocytes contain well visualized cytoplasmic vacuoles and less number of liposomes compared to the rats exposed to 10-day $+G x$ acceleration. Lipid droplets are located in close contact with the mitochondria, with enlightened matrix in most of them. Also, expansion of the endoplasmic reticulum cisterns near the nucleus and liposomes is observed.

There is a slight blurring of the border between zona fasciculata and reticularis in the adrenal cortex. Unlike previous series of experiments, the relative area of zona reticularis decreases by only $23.88 \%$ compared to the control $(p<0.05)$. Endocrinocytes in this zone less tightly contact with each other. Cells are characterized by large round nuclei (Table 2), enlightened cytoplasm, and mitochondria with vesicular changed cristae. 


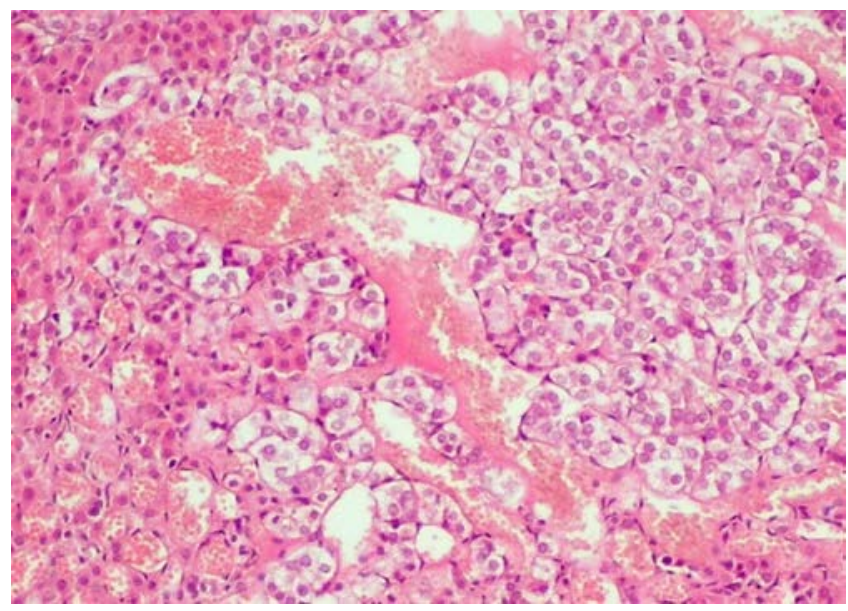

Figure 4. Pronounced hyperemia and vasodilatation of the microvasculature in the adrenal medulla in juvenile rat under the systematic exposure to 10 -day $+G x$ acceleration. H\&E. x400.

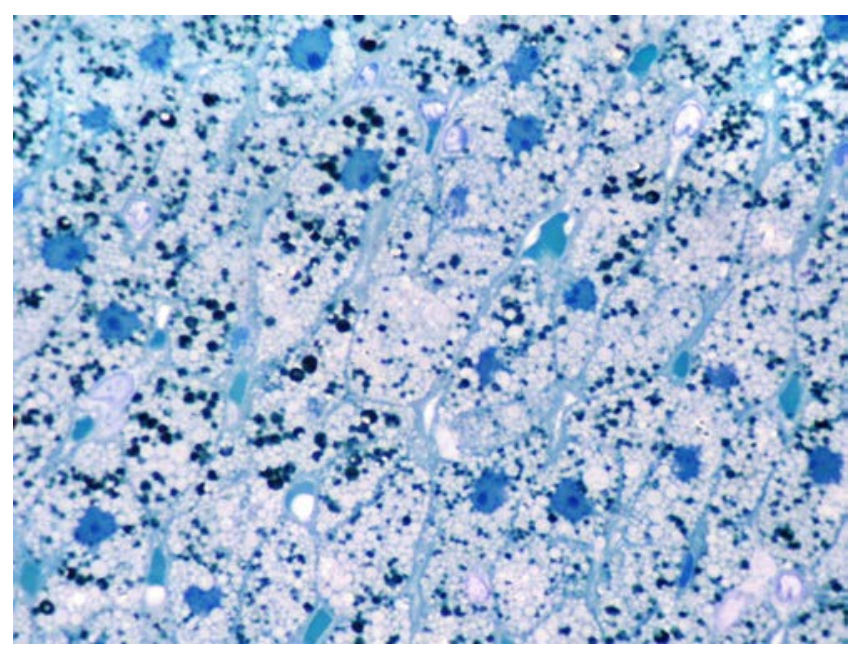

Figure 5. Pronounced increase in number of dark corticocytes with a lot of liposomes in their cytoplasm. Zona fasciculata of adrenal cortex in juvenile rat under the systematic exposure to 30 -day $+G x$ acceleration. Semithin section. Toluidine blue. $\mathbf{x} 1000$.

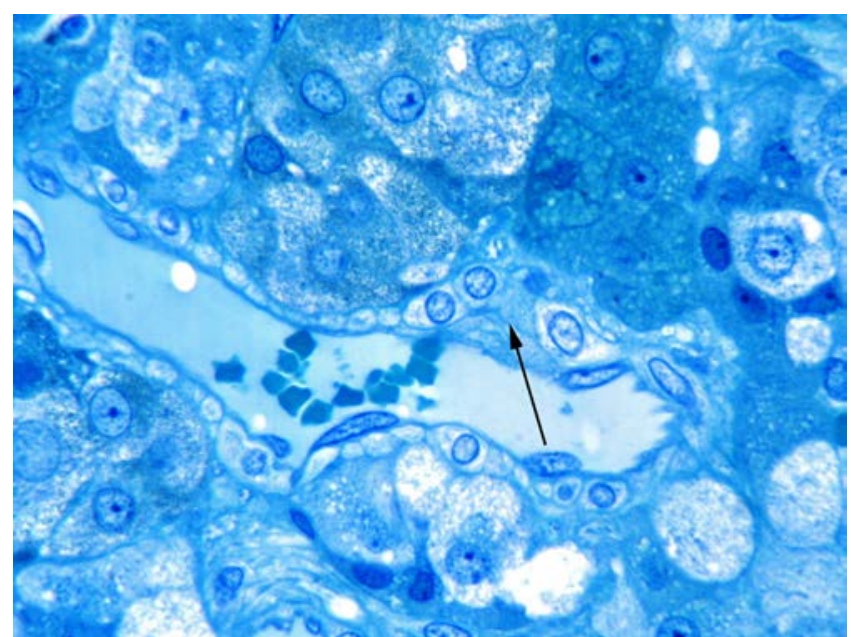

Figure 6. Focal thickening of the basement membrane and perivascular increase of the fibroblastic cells (arrow). Adrenal medulla in juvenile rat under the systematic exposure to 30 -day $+G x$ acceleration. Semithin section. Toluidine blue. $x 1000$.
Adrenal medulla looks edematous, though, at the same time, its relative area in the parenchyma of the adrenal gland is less than the control value (decrease by $25.81 \%$; $p<0.05$ ). Some plethoric microcirculatory vessels are characterized by the thickened basement membrane. Proliferation of the connective tissue and fibrosis is seen at perivascular spaces. Foci of old hemorrhages are present at the intercellular substance (Figure 6).

Chromaffin cells have the ultrastructural signs of inhibition of synthesis and secretion of "acute" stress hormones (catecholamines), such as a destruction of the mitochondria cristae, Golgi complex and decrease in number and size of secretory granules. Occasionally, there are macrophages with inclusions of phagocyted chromaffin cells debris in their cytoplasm.

Unlike the previous two (10- and 30-day) series of experiments, 45-day exposure to $+G x$ acceleration results in decrease in the relative weight of the adrenal glands of experimental rats by $13.77 \%(p<0.05)$. As a matter of interest, changes in the adrenal parenchyma are minor compared to the control in terms of the areas of the adrenal cortex and medulla. Thus, the relative area of the cortex insignificantly reduces by $1.19 \%$ and area of the medulla increases by $9.31 \%(p<0.05)$ compared to the control (Table 1).

Histologically, mild circulatory disorders that reflect compensatory-adaptive reactions are observed. Some of the blood vessels are dilated and plethoric, and some are characterized by stasis and sludge-phenomenon. The capillary basement membrane is thickened unevenly due to focal excess of perivascular located connective tissue fibers. Same fibrotic changes can be seen in the capsule.

Adrenal parenchyma still has mild edema with increased content of the connective tissue component with focal thickening of the connective tissue bands between endocrinocytes. Redistribution of zone areas is observed. Compared to the control, the relative areas of zona glomerulosa and zona reticularis increase by $37.42 \%$ and $15.00 \%$, respectively, while area of zona fasciculata decreases by $7.84 \%$ (all $p<0.05$ ) (Table 1 ).

Adrenocorticocytes at zona glomerulosa contain large nuclei, and their area as compared to the control increase by $41.46 \%$ $(p<0.05)$ (Table 2).

Zone fasciculata is characterized by non-significant increase (by $2.50 \%$ ) in the density of spongiocytes population, while significant reducing area of their nuclei (by $16.74 \%$; $p<0.05$ ). The percentage of light and dark cells is close to the control values (Tables 2 and 3). Content of lipid inclusions in their cytoplasm does not significantly differ from the control (Table 4). At the same time, mitochondria destruction persists with a various severity. Cytoplasmic matrix is compacted, and there are a significant number of contacts between the mitochondria and lipid droplets (Figure 7).

Cells in zona reticularis look smaller in size than in the control, which is confirmed by morphometry: the average size of their nuclei is reduced by $25.47 \%(p<0.05)$.

Medulla chromaffin cells are also characterized by reduction of the nucleus area (by $25.37 \% ; \mathrm{p}<0.05$ ). In addition, compared to the previous series (30-day exposure), their cytoplasm contains increased number of the secretory granules. 


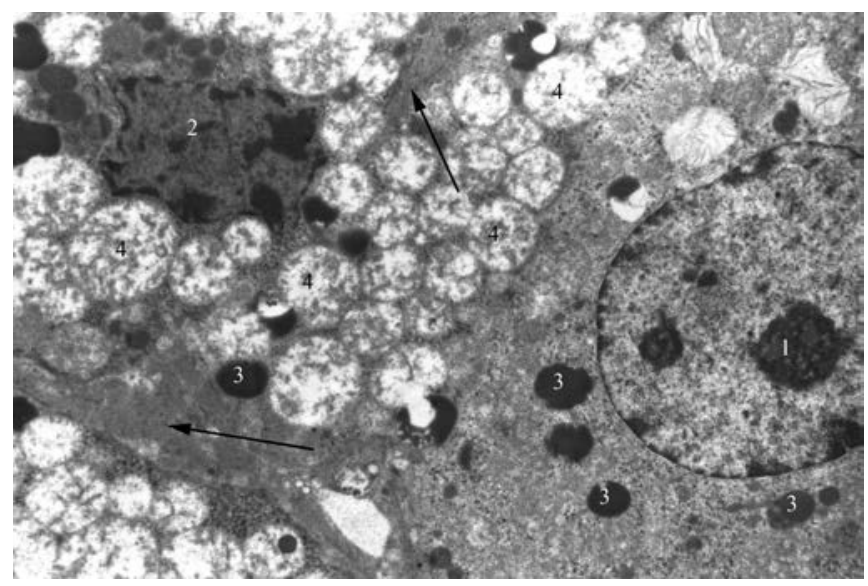

Figure 7. Dark and light endocrinocytes of adrenal zona fasciculata in juvenile rat under the systematic exposure to 45-day $+G x$ acceleration. TEM. x4800. 1 - light endocrinocyte nucleus; 2 - dark endocrinocyte nucleus; 3 - liposomes; 4 -mitochondria; arrows - focal thickening of the connective tissue between the cells.

\section{Discussion}

It is well known that body's response to gravitational exposure is accompanied by significant activation of sympathoadrenal and pituitary-adrenal systems typical for the stress reaction [11, 12]. At the same time, the nature, severity and reversibility of changes depend on many factors, including the characteristics of the hypergravity influence, such as its duration (acute or chronic), multiplicity (single or multiple), magnitude (low or high) and direction.

In terms of duration of gravitational overloads, acute stress caused by short but strong hypergravitational factor involves adaptive pituitary hormones and, in particular, adrenocorticotropic hormone (ACTH), which in turn triggers increased synthesis of corticosteroids in adrenal glands [12, 13]. However, hypergravity-induced adrenocortical reaction in case of chronic influence changes during physiological adaptation. Prolonged gravitational accelerations cause reduction of ACTH in plasma that may be due to possible impact of the feedback mechanism caused by the significant level of glucocorticoids secreted by the adrenal glands under the stress condition. For example, experiments on rats exposed to $4.5 \mathrm{G}$ acceleration showed increase in cortisol level during the 1st hour of exposure, with its gradual decrease subsequently $[14,15]$. Also, it was demonstrated that zona fasciculata after the acute exposure to gravitational overloads characterized by the signs of high secretory activity, while after long-term exposure for 13 weeks and 26 weeks dramatic inhibition of hormone synthesis and secretion was observed [15]. Thus, such changes in hormone production should be considered as an endocrine mechanism representative of the divergence of plastic processes and energy metabolism with prolonged and constant hypergravity exposure.

In the context of magnitude of hypergravity, according to the literature data, histomorphological changes in the adrenal glands are seen mostly at the acceleration level over $8 \mathrm{G}$. Thus, it was shown that exposure to gravitational overloads $25 \mathrm{G}$ in transverse direction for 10 minutes leads to the development of edema, hemorrhages in the adrenal cortex, and reduced lipid content. Similar, destructive changes in mitochondria ultrastructure in the secretory cells of adrenal cortex resulted in decrease in the synthesis of corticosteroids $[8,15]$.
In general, the presented literature data have both theoretical and practical interest, and provide an idea of the possible nature of the adrenal response to hypergravity-induced stress, but these data are incomplete and based on a variety of experimental models of hypergravity effects, which makes their comparison difficult. Also, there is practically no comprehensive coverage of the morphological changes in the adrenal glands at different levels of structural organization, as well as features of morphological and functional changes in the adrenal glands in the age aspect.

On the other hand, the present experimental study, although it is not beyond certain limitations, is an extended study of the morphological features of the adrenal glands under the hypergravity exposure with significant magnitude $(9 \mathrm{G})$ in juvenile animals, with a clear separation in the experiment duration $(10,30$ and 45 days) to determine the dynamics of hypergravity-induced changes.

Thus, the reaction of the adrenal glands following 10-day exposure was characterized by severe stress-induced activation, as evidenced by significantly increased (in comparison with the control, by 2.3 times) relative adrenal mass, increased cortex relative area (by $8.14 \%$ ) and the predominance of functionally active light adrenocortical cells. Lipid inclusions in the spongiocytes cytoplasm demonstrate a possible violation of recycling of the unused cholesterol esters due to altered mitochondrial function, and on the other hand may show a trend towards normalization of the functional state of the cells and increasing their hormonal function [16]. Along with this, adrenal medulla was characterized by the morphological signs of "wear" of the chromaffin cells and decreasing in their function.

With an increase in the duration of the experiment to 30 days, as well as at the previous observation period, the adrenal glands were characterized by an increase in the relative weight (compared to control by more than 2.5 times) and expansion of the zona fasciculata (by 6.27\%). At the same time, unlike 10-day experiment, the density of the cellular distribution in this zone was increased (by $31.39 \%$ ), and most of spongiocytes were dark cells with high nuclear-cytoplasmic ratio and high content of cytoplasmic lipid vacuoles. According to the literature data, such observations may be considered as a morphological evidence of active functioning of the stress-induced hormonal system $[16,17]$. On the other hand, an increment of the direct negative impact was manifested in congestion of the microvasculature and observed degenerative changes in endocrinocytes (mostly, in secretory cells of the adrenal medulla). Chromaffin cells were present with the morphological signs of inhibition of synthesis and secretion of catecholamines (destruction of the mitochondria cristae and Golgi apparatus, reduction in size and number of the secretory granules).

Following 45 days of the experiment, unlike the previous two (10 and 30 days) series, adrenal glands, which functional activity is a key factor in body's response to the stress and adaptation, reacted in sufficiently balanced manner. It was manifested in decreasing the relative weight of the organ (by $13.77 \%$ compared to control) in the absence of significant variations in the ratio of cortex and medulla. On the background of the expansion of the relative area of zona glomerulosa and reticularis there was somewhat reduced zona fasciculata (by $7.84 \%$ ). The ratio of dark and light spongiocytes, as well as their lipid cytoplasm inclusions showed no significant differences compared to the control, despite presence of the ultrastructural degeneration changes. In turn, 
chromaffin cells, as compared with the previous series of experiments, were characterized by the increasing content of the secretory granules. In our opinion, these changes in the adrenal architectonic along the moderate structural and functional transformation of the cortical and medullary endocrinocytes can be considered as a manifestation of "systemic structural trace", formed as a result of the repeated hypergravity exposure. The overall reaction of the adrenal cortex appears as mild in contrast to two previous series of experiments, and, at first glance, the lower functional activity of the organ actually does not correspond to the ongoing stress. However, review of the literature data suggests several possible mechanisms for implementing such changes, including depletion of the functional reserves (the "overtraining" effect), as well as the inhibition of excessively activated cortex by other humoral factors $[15,17]$.

In conclusion, it can be assumed that the systematic effect of the hypergravity accelerations results in significant changes in the structural and functional organization of adrenal glands, changing the balance of the endocrine stress response. In particular, despite the limited data in the literature, this is confirmed by the absence of a number of physiological changes in adrenalectomized rats exposured to $+10 \mathrm{Gz}$ acceleration ( $+\mathrm{Gz}$ acceleration is acceleration acting in head to feet axis) [18]. These changes can be grouped into: stress-induced activation of the adrenal cortex; hemodynamic microvasculature disturbances, leading to hypoxia and tissue alteration; and implementation of the adaptive reactions that may occur in an adequate or inadequate extent.

\section{Conclusion}

The observed structural transformations in the adrenal glands under the hypergravity exposure can be considered as the adaptive responses to stress and hemodynamic changes. The significant hemodynamic changes combined with stress-induced morphofunctional transformations in the form of the destructive ultrastructural changes were most pronounce changes in the adrenal glands. Increased duration of the experiment up to 45 days resulted in the stabilization of morphofunctional organisation of the adrenal glands, limited to the "systemic structural trace" and possible depletion of the functional reserves.

\section{Study Limitations}

The present experimental study was preformed on juvenile Wistar rats exposed to multiple episodes of $9 \mathrm{G}$ hypergravity over a period of 10, 30 and 45 days. Due to this, this study is limited only to those experimental conditions that have been modeled. Also, there are reasonable limitations related to the extrapolation of the obtained data to the humans, taking into account the species differences in the stress reaction and adaptive reserves.

\section{Conflict of interest}

The authors declare that they have no conflict of interest.

\section{Ethical Approval}

All applicable international, national, and/or institutional guidelines for the care and use of animals were followed. All procedures performed in the study involving animals were in accordance with the ethical standards of the institution or practice at which the study was conducted.

\section{References}

1. Ogawa Y, Yanagida R, Ueda K, Aoki K, Iwasaki K-I. The relationship between widespread changes in gravity and cerebral blood flow. Environ Health Prev Med 2016; 21: 186-192. https://doi.org/10.1007/s12199-016-0513-7.

2. Pendergast DR, Olszowka A, Fahri LE. Cardiovascular and pulmonary responses to increased acceleration forces during rest and exercise. Aviat Space Environ Med 2012; 83(5): 488-495. https://doi.org/10.3357/ASEM.3127.2012.

3. Kotovskaya AR. Symptoms of negative effects cumulation in humans and animals under the action of G-loads of varying direction in context of aviation and space flights. Aviakosm Ekolog Med 2015; 49(6): 14-18. Russian. https://www.ncbi.nlm.nih.gov/pubmed/26934785.

4. Mikuliszyn R, Zebrowski M, Kowalczuk K. Centrifuge training program with "push-pull" elements. Aviat Space Environ Med 2005; 76(5): $493-$ 495. https://www.ncbi.nlm.nih.gov/pubmed/15892550.

5. Petrak J, Mravec B, Jurani M, Baranovska M, Tillinger A, Hapala I, et al. Hypergravity-induced increase in plasma catecholamine and corticosterone levels in telemetrically collected blood of rats during centrifugation. Ann N Y Acad Sci 2008; 1148: 201-208. https://doi.org/10.1196/annals.1410.060.

6. Sukhoterin AF, Pashchenko PS, Plakhov NN, Zhuravlev AG. Role of the sympathadrenomedullary system in formation of pilot's adaptation to flight loads. Aviakosm Ekolog Med 2015; 49(5): 49-53. Russian. https://www.ncbi.nlm.nih.gov/pubmed/26738308.

7. Gueguinou N, Bojados M, Jamon M, Derradji H, Baatout S, Tschirhart E, et al. Stress response and humoral immune system alterations related to chronic hypergravity in mice. Psychoneuroendocrinology 2012; 37(1): 137-147. https://doi.org/10.1016/j.psyneuen.2011.05.015.

8. Krasnov IB, Alekseev EI, Loginov VI. Role of the endocrine glands in divergence of plastic processes and energy metabolism in rats after extended exposure to hypergravity: cytologic investigation. Aviakosm Ekolog Med 2006; 40(3): 29-34. Russian. https://www.ncbi.nlm.nih.gov/pubmed/17193966.

9. Schneider CA, Rasband WS, Eliceiri KW. NIH Image to ImageJ: 25 years of image analysis. Nature Methods 2012; 9(7): 671-675. https://www.ncbi.nlm.nih.gov/pubmed/22930834.

10. Justel A, Peña D, Zamar R. A multivariate Kolmogorov-Smirnov test of goodness of fit. Statistics \& Probability Letters 1997; 35(3): 251-259. https://doi.org/10.1016/S0167-7152(97)00020-5.

11. Goodall MC. Sympathoadrenal response to gravitational stress. J Clin Invest 1962; 41(2): 197-202. https://doi.org/10.1172/JCl104471.

12. Macho L, Kvetnansky R, Fickova M, Popova IA, Grigoriev A. Effects of exposure to space flight on endocrine regulations in experimental animals. Endocr Regul 2001; 35: 101-114. https://www.ncbi.nlm.nih.gov/pubmed/11563939.

13. Horii A, Mitani K, Masumura C, Uno A, Imai T, Morita Y, et al. Hippocampal gene expression, serum cortisol level, and spatial memory in rats exposed to hypergravity. J Vestib Res 2017; 27(4): 209215. https://doi.org/10.3233/VES-170521.

14. Vasil'yev PV, Kotovskaya AR. Chapter 5. Prolonged linear and radial accelerations. In: Gazenko OG, Melvin C. Foundations of space biology and medicine. Joint USA/USSR Publication; Vol. 2, Part 1: Ecological and physiological bases of space biology and medicine, 1975: 163 213. Russian. https://ntrs.nasa.gov/archive/nasa/casi.ntrs.nasa.gov/19760019721.pdf.

15. Pashchenko PS, Korneva EF, Kamaldinova AT, Egorov VA. Structural and functional transformations in the zona fasciculata of the adrenal cortex during experimental modeling of gravitational stress. Bulletin of the Russian Military Medical Academy 2017; S3: 127-128. Russian. https://elibrary.ru/item.asp?id=32334916.

16. Rosol TJ, Yarrington JT, Latendresse J, Capen CC. Adrenal gland: structure, function, and mechanisms of toxicity. Toxicologic Pathology 2001; 29(1): 41-48. https://doi.org/10.1080/019262301301418847. 
17. Koldesheva EV. Ultrastructural equivalents of adaptive reorganization of adrenal cortex under extremal factors. Bulletin of Siberian Branch of Russian Academy of Medical Sciences 2008; 28(6): 139-144. Russian. https://elibrary.ru/item.asp?id=12365303.

18. Na K, Kim H-S. Adrenalectomy abolishes hypergravity-induced gastric acid hyposecretion. Oncotarget 2017; 8(19): 30700-30705. https://doi.org/10.18632/oncotarget.15408.

\section{Authors:}

Gennady A. Moroz - MD, DSc, Professor, Head of the Department of Medical Physical Culture, Sports Medicine and Physiotherapy with a course of Physical Culture, Medical Academy n.a. S.I. Georgievsky, V.I. Vernadsky Crimean Federal University, Simferopol, Russia. http://orcid.org/00000001-7145-6564.

Maxim A. Kriventsov - MD, DSc, Professor, Head of the Department of Pathological Anatomy with a Sectional Course, Medical Academy n.a. S.I. Georgievsky, V.I. Vernadsky Crimean Federal University, Simferopol, Russia. http://orcid.org/0000-0001-5193-4311.

Sergey A. Kutia - MD, DSc, Professor, Head of the Department of Medical Biology, Medical Academy n.a. S.I. Georgievsky, V.I. Vernadsky Crimean Federal University, Simferopol, Russia. http://orcid.org/0000-0002-11454644 . 